Revue internationale P.M.E.

Économie et gestion de la petite et moyenne entreprise

\title{
L'analyse stratégique et ses outils de gestion: application dans l'entreprise agricole au Québec
}

\section{Rémi Lambert et Stéphane Maisonnas}

Volume 12, numéro 1-2, 1999

URI : https://id.erudit.org/iderudit/1008648ar

DOI : https://doi.org/10.7202/1008648ar

Aller au sommaire du numéro

Éditeur(s)

Presses de l'Université du Québec

ISSN

0776-5436 (imprimé)

1918-9699 (numérique)

Découvrir la revue

Citer cet article

Lambert, R. \& Maisonnas, S. (1999). L'analyse stratégique et ses outils de gestion: application dans l'entreprise agricole au Québec. Revue internationale P.M.E., 12(1-2), 15-30. https://doi.org/10.7202/1008648ar
Résumé de l'article

Le présent article livre les résultats d'une étude sur la gestion stratégique dans le secteur agricole du Québec. L'objectif de cette étude visait à définir, à la lumière de ce qui se fait dans la petite et moyenne entreprise (PME) industrielle, une démarche de gestion stratégique adaptée pour les gestionnaires d'entreprises agricoles. Nous voulions aussi déterminer les outils de gestion qui rendraient cette démarche opérationnelle. Ainsi, des entrevues avec les gestionnaires agricoles ont permis de valider la démarche proposée et de déterminer les outils qui sont actuellement utilisés en agriculture et qui servent à la démarche stratégique. De plus, nous avons constaté que plusieurs outils utilisés par la PME sont absents de la gestion agricole. À notre avis, l'adoption de ces outils favoriserait j'utilisation accrue de la gestion stratégique en agriculture. 


\title{
L'analyse stratégique et ses outils de gestion: application dans l'entreprise agricole au Québec
}

\author{
Rémi LAMBERT \\ Université Laval
}

Stéphane MAISONNAS

École des Hautes Études commerciales de Montréal

\section{MOTS CLÉS}

\section{Gestion stratégique - Agriculture - Gestion de la PME Outils de gestion}

\begin{abstract}
RÉSUMÉ
Le présent article livre les résultats d'une étude sur la gestion stratégique dans le secteur agricole du Québec. L'objectif de cette étude visait à définir, à la lumière de ce qui se fait dans la petite et moyenne entreprise (PME) industrielle, une démarche de gestion stratégique adaptée pour les gestionnaires d'entreprises agricoles. Nous voulions aussi déterminer les outils de gestion qui rendraient cette démarche opérationnelle. Ainsi, des entrevues avec les gestionnaires agricoles ont permis de valider la démarche proposée et de déterminer les outils qui sont actuellement utilisés en agriculture et qui servent à la démarche stratégique. De plus, nous avons constaté que plusieurs outils utilisés par la PME sont absents de la gestion agricole. À notre avis, l'adoption de ces outils favoriserait l'utilisation accrue de la gestion stratégique en agriculture.
\end{abstract}

\section{ABSTRACT}

This article provides the results of a study on strategic management in the Quebec agricultural sector. The main objective was to identify, in the light of what industrial small and medium-sized enterprises (SME) are doing, a strategic management approach suitable for farm managers. We also wanted to identify tools that would

\section{LES AUTEURS}

Rémy Lambert est professeur au Département d'économie agroalimentaire et des sciences de la consommation et directeur du Centre de recherche en économie agroalimentaire, Université Laval, Québec, Canada.

Stéphane Maisonnas, ancien étudiant de deuxième cycle en économie rurale, Université Laval, poursuit présentement des études de doctorat aux HEC de Montréal, Québec, Canada. 
make this approach operational. Interviews with farm managers contributed to validate the proposed approach and to identify which tools already used in agricultural firms are appropriate for the strategic approach. In addition, we observed that several tools used by SMEs were missing in farm management. Therefore, using them would promote strategic management in agriculture.

\section{RESUMEN}

Este artículo presenta los resultados de un estudio sobre la gestión estratégica en el sector agrario de Quebec. El objetivo de este estudio es establecer, a la luz de lo que se hace con la pequeña y mediana empresa (PyME) industrial, un enfoque de gestión estratégica adaptado a los responsables de empresas agrícolas, así como identificar las herramientas de gestión que permitirían validar el enfoque propuesto. De este modo, las entrevistas con los gestores agrícolas han permitido validar el enfoque y determinar las herramientas que se emplean actualmente en el sector y que se emplean en el enfoque estratégico. Además, varias herramientas utilizadas por las PyMEs no se emplean en la gestión agraria. Entendemos que la adopción de estas herramientas favorecería un mayor uso de la gestión estratégica en la agricultura.

\section{ZUSAMMENFASSUNG}

Der vorliegende Artikel liefert die Resultate einer Studie über die strategische Unternehmungsführung im Agrarsektor in Quebec.

Ziel dieser Studie war die Definition der strategischen Führung für eine Agrarunternehmung, indem die in KMU angewandten Methoden angepasst wurden. Im weiteren wurde versuchte jene Führungsinstrumente zu bestimmen, die eine Operationalisierung dieser Vorgehensweise ermöglichte. Interviews mit Agrarunternehmern erlaubten die Validierung der vorgeschlagenen Vorgehensweise. Gleichzeitig konnten die Instrumente festgelegt werden, die momentan in der Agrarwirtschaft verwendet werden und der strategischen Führung dienen. Wobei zu beachten ist, dass nicht alle in den KMU verwendeten Instrumente bei einer Agarunternehmung eingesetzt werden können. Aber die Billigung der verwendeten Instrumente favorisierte eine erhöhte strategische Führung in der Agrarwirtschaft.

\section{Introduction}

Jusqu'à présent, la gestion agricole au Québec a principalement porté sur la gestion des opérations. En effet, en étant assuré de vendre sa production, en répondant à une demande en expansion et en disposant des capacités de production appropriées, le producteur a souvent eu pour unique préoccupation celle de maximiser son revenu en surmontant certaines contraintes d'ordre physique ou matériel.

Cependant, les hypothèses de base ont évolué : le progrès technologique a conduit à la surproduction ; les marchés ont été progressivement saturés ; les contraintes environnementales se sont de plus en plus fait sentir. De surcrôit, 
l'internationalisation annonce une mondialisation des marchés par une plus grande ouverture des frontières ! C'est dans ce nouvel environnement décisionnel que les entreprises agricoles doivent désormais être gérées. Or, la gestion des opérations ne suffit plus à répondre à ces exigences.

Il convient alors de poser la question suivante : «Peut-on trouver un moyen d'améliorer la capacité de gestion des producteurs et des productrices agricoles dans ce nouvel environnement et, par le fait même, augmenter leur rentabilité et leur compétitivité sur les marchés nationaux et internationaux?»

À cette question générale de recherche, les chercheurs ont répondu en proposant l'approche de gestion stratégique, telle qu'elle est utilisée dans les petites et moyennes entreprises (PME) du monde industriel (Westgren, Sonka et Litzenberg, 1988; Attonaty et Soler, 1992 ; Harling, 1992; Lambert, Calkins et Michaud, 1992). Non seulement l'atteinte des objectifs est plus efficace grâce à cette approche (Lambert, Calkins et Michaud, 1992), mais elle est également un facteur de réussite pour les entreprises agricoles comme elle l'a été pour les entreprises industrielles (Harling, 1992). Malgré ce besoin et les réponses qu'elle pourrait apporter, les auteurs (Harling, 1992 ; Lambert, Calkins et Michaud, 1992) déplorent que cette démarche ne soit pas plus fréquemment observée en agriculture. Par exemple, seulement $12 \%$ des producteurs horticoles de la région de Québec pratiquent un type de gestion se rapprochant de la gestion stratégique (Lambert, Calkins et Michaud, 1992).

Selon les mêmes auteurs, ce faible niveau d'adoption s'expliquerait principalement par l'absence d'outils appropriés. En effet, parmi les initiatives en gestion stratégique pour l'entreprise agricole menées au Québec, aucune ne propose les outils d'analyse qui rendraient le processus opérationnel. Afin de mieux cerner l'action stratégique au sein de l'entreprise agricole et la rendre opérationnelle, nous proposons d'identifier les outils d'aide à la prise de décision qui sont absents de la pratique actuelle et qui contribueraient à rendre cette démarche de gestion opérationnelle en agriculture. Nous croyons que cela représente un pas important vers l'adoption de la gestion stratégique en agriculture et vient compléter les actions actuelles en vue de promouvoir le concept.

L'objectif général de cette étude est donc d'analyser, dans un cadre de gestion stratégique, les outils actuellement utilisés en agriculture au Québec et de vérifier la pertinence, pour ce secteur, de recourir aux outils de gestion de la PME non agricole.

\section{Méthodologie}

Afin d'atteindre l'objectif de cette recherche, un cadre d'analyse stratégique a été mis au point, puis validé auprès de conseillers agricoles de la grande région de Québec. Ce cadre d'analyse a été établi grâce à une revue de littérature portant sur 
l'analyse stratégique dans les PME industrielles et agricoles. La démarche proposée se compose de trois phases principales et elle est illustrée aux figures 1 (Phase I) et 2 (Phase II et III). Pour obtenir de plus amples détails sur la démarche proposée, le lecteur est invité à consulter Lambert et al. (1995).

L'enquête, comme stratégie générale de vérification, et plus particulièrement les entrevues, comme moyen de collecte de l'information, ont été retenus. La population à l'étude a été définie comme étant celle des gestionnaires agricoles de la grande région de Québec (Région agricole 02). Six groupes de personnes ont été rejoints : les conseillers en gestion du ministère de l'Agriculture, des Pêcheries et de l'Alimentation du Québec (MAPAQ), de l'Union des producteurs agricoles (UPA), de la Société de financement agricole du Québec et de la Société de crédit agricole du Canada, des institutions financières et des syndicats de gestion agricole (SGA).

Nous avons d'abord choisi les personnes à interroger et nous avons pris les mesures nécessaires pour obtenir un rendez-vous avec elles. Pour les SGA, les quatre conseillers de la région ont été joints. Pour l'UPA, une seule personne a été identifiée comme conseiller de gestion. Pour les autres groupes, une personnecontact a établi à notre demande une liste des conseillers en gestion de son institution. C'est à partir de cette liste qu'ont été choisies les personnes à enquêter. Pour certaines banques ne possédant pas de telle liste, les contacts ont été établis au hasard des succursales susceptibles de s'occuper de gestion agricole dans la région.

Lors de l'entrevue, nous avons demandé aux conseillers d'évaluer la démarche proposée ; les réactions ont été très positives et de légères modifications ont été apportées à la lumière de leurs commentaires. L'entrevue a aussi servi à déterminer les pratiques et les besoins des gestionnaires agricoles en matière d'analyse stratégique. Cette évaluation a été faite à l'aide d'une étude de cas présentée aux conseillers où ils avaient à résoudre un problème de gestion en désignant les étapes et les outils utilisés. Les entrevues semi-structurées étaient conduites individuellement, ce qui a permis non seulement de connaître les pratiques et les besoins des gestionnaires agricoles, mais aussi de recueillir leur avis sur la démarche de gestion stratégique et les outils que nous leur proposions.

Le nombre de personnes à joindre par catégorie s'est déterminé de lui-même. Quand la catégorie était peu importante, nous avons pris contact avec tous ses membres. Par contre, si la catégorie comportait un nombre élevé de personnes, comme c'était le cas pour les conseillers de financement, il nous était impossible de déterminer a priori le nombre de personnes à joindre. Nous cessions les entretiens dans cette catégorie lorsqu'il y avait saturation de l'information, c'est-à-dire lorsque les enquêtés donnaient la même information. 
FIGURE 1

Phase I de la démarche de gestion stratégique

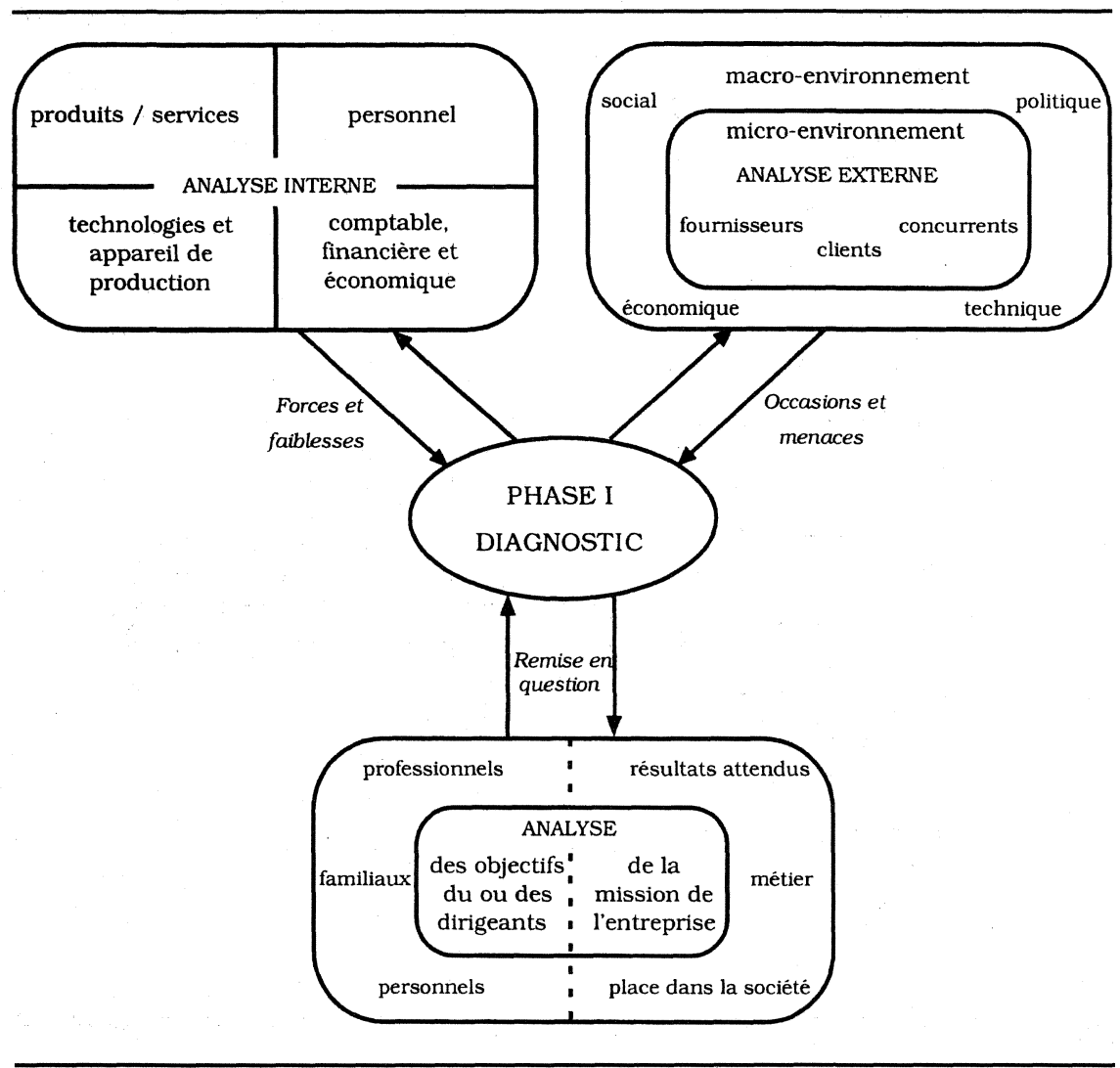

\section{Analyse des résultats}

Le tableau 1 présente les institutions, le nombre de personnes jointes par institution, ainsi que les raisons justifiant l'arrêt des enquêtes. Au bout de 15 entretiens, la saturation de l'information était atteinte pour l'ensemble des enquêtés, toutes catégories confondues. La démarche de gestion stratégique proposée est apparue familière aux conseillers. Selon eux, les concepts abordés ressemblent à ceux proposés par d'autres auteurs et constituent une démarche dont ils ont déjà entendu parler. Toutefois, ils jugent que cette démarche présente l'avantage d'aborder les concepts de façon plus claire. De plus, par rapport à ce qu'ils connaissent de la gestion stratégique, elle a le mérite de mieux structurer la réflexion. 
FiguRE 2

\section{Phase II et III de la démarche de gestion stratégique}

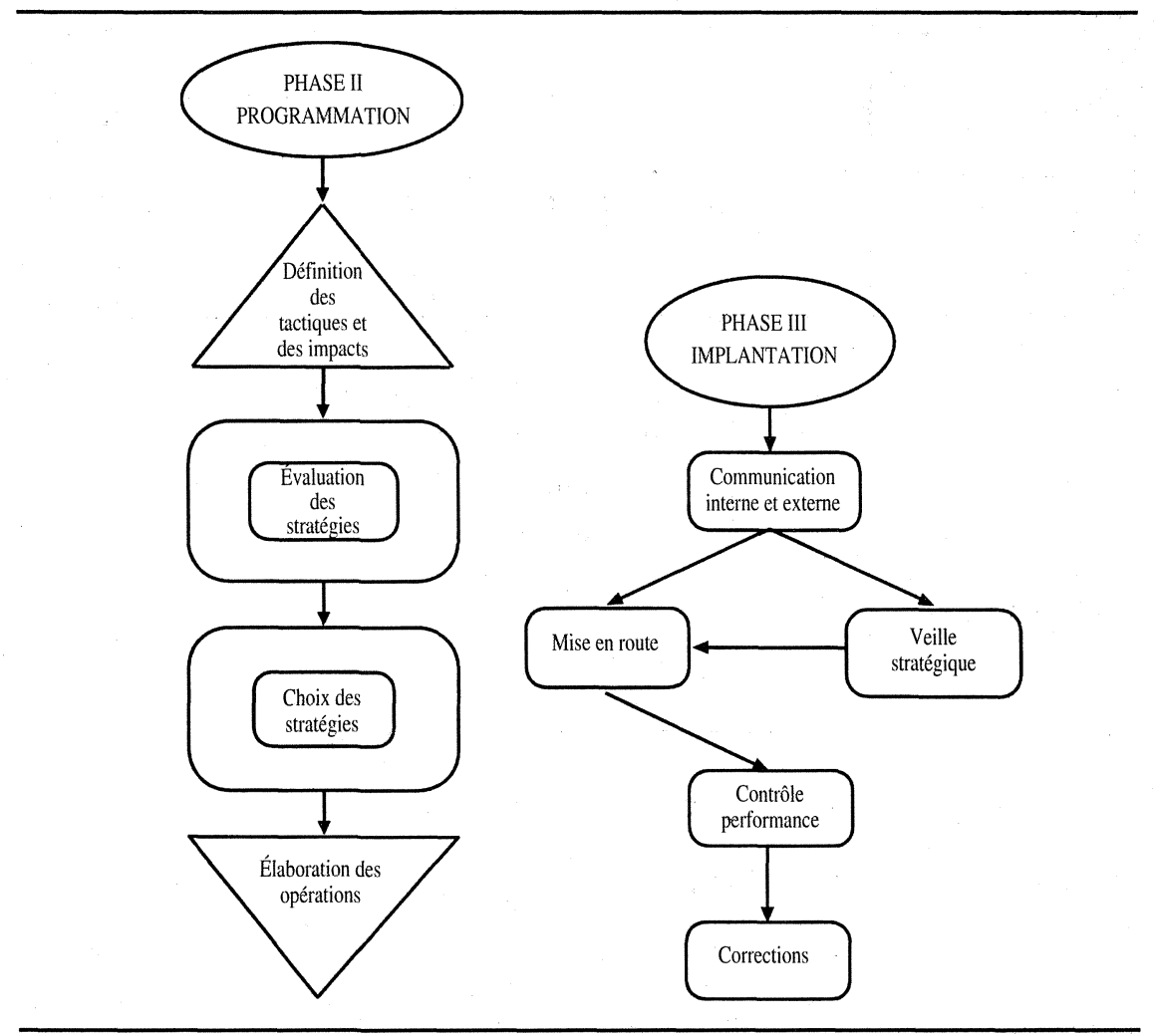

TABLEAU 1

Population enquêtée

\begin{tabular}{lcl}
\hline $\begin{array}{l}\text { Origine } \\
\text { de la population }\end{array}$ & $\begin{array}{c}\text { Nombre d'intervenants } \\
\text { joints }\end{array}$ & $\begin{array}{l}\text { Raisons de l'arrêt } \\
\text { des enquêtes }\end{array}$ \\
\hline Syndicat de gestion & 3 & Toute la population joignable \\
Société de financement agricole & 3 & Saturation de l'information \\
Société de crédit agricole & 2 & Saturation de l'information \\
MAPAQ & 2 & Toute la population joignable \\
UPA & 1 & Toute la population \\
Institutions financières & 4 & Saturation de l'information \\
\hline Total & $\mathbf{1 5}$ & \\
\hline
\end{tabular}


Les répondants affirment, en outre, que cette démarche pourrait aider à relever les nouveaux défis de l'entreprise agricole. Ils estiment en revanche qu'elle n'est encore ni appliquée, ni applicable chez la plupart des agriculteurs. Ceci n'est pas une caractéristique de la démarche en soi, mais le reflet d'un manque de formation et de sensibilisation chez les producteurs. Selon les conseillers, les agriculteurs avant-gardistes sont peut-être prêts à entreprendre une telle démarche, mais ils représentent une minorité des entreprises agricoles.

Selon divers auteurs, les critères qu'une bonne démarche de gestion stratégique doit respecter sont les suivants :

- la flexibilité, contraire de la formalité et de la rigidité (D'Amboise, 1986);

- la proximité avec la réalité opérationnelle (Mintzberg, 1994) qui se caractérise, en agriculture, par la fragilité et la forte dépendance à l'environnement et la petite taille économique avec des domaines de compétence très délimités (Attonaty et Soler, 1992);

- l'adaptabilité au plus grand nombre d'entreprises (Attonaty et Soler, 1992);

- l'apport de solutions pour aider les dirigeants à atteindre leurs objectifs (D'Amboise, 1986);

- l'exhaustivité, c'est-à-dire qu'elle doit aborder tous les points nécessaires (D'Amboise 1994).

Aux yeux des gestionnaires agricoles, la démarche proposée de gestion stratégique satisfait tous ces critères. Ainsi, les conseillers avaient à déterminer, sur une échelle de un (pas du tout) à cinq (totalement) si le critère était respecté. L'analyse des notes données par les conseillers sur les différents critères suggérés, leur moyenne, la variance et le coefficient de variation est présentée au tableau 2. Les notes sont toutes supérieures à 3,7 , ce qui est une bonne moyenne. De plus, les variations autour de cette moyenne vont de 1,41 à 0,41 , soit un coefficient de variation entre $31 \%$ et $11 \%$. Les notes les plus faibles visent la flexibilité, la proximité avec la réalité interne de l'entreprise et la gestion agricole, et l'exhaustivité. Les trois premières révèlent que la réalité actuelle de la gestion agricole est loin de la démarche de gestion stratégique élaborée, comme l'ont souligné Lambert, Calkins et Michaud (1992). Toutefois, les enquêtés sont d'avis qu'il faut s'orienter vers ce type de gestion. Donc, la faiblesse de ces critères pourrait mieux s'expliquer par un manque de sensibilisation et de formation des agriculteurs que par le fait qu'il s'agit d'une démarche déconnectée de la réalité. L'exhaustivité pourrait être accrue en précisant les outils d'analyse ou les postes à étudier; pour ce faire, il faudra donc être plus clair dans la présentation de la démarche. Les notes moyennes sont à 3,87 . Il s'agit de l'adaptabilité, de la proximité avec la réalité externe de l'entreprise et la solution aux problèmes actuels. Donc, cette démarche, si elle respecte le critère d'adaptabilité, peut être implantée dans n'importe quel type d'exploitation. De plus, 
TABLEAU 2

Notes d'évaluation de la démarche

\begin{tabular}{lcccccccc}
\hline Critères & $\mathbf{1}$ & $\mathbf{2}$ & $\mathbf{3}$ & $\mathbf{4}$ & $\mathbf{5}$ & Moyenne & Variance & $\begin{array}{c}\text { Coefficient } \\
\text { de variation }\end{array}$ \\
\hline $\begin{array}{l}\text { Flexible } \\
\text { Adaptable }\end{array}$ & 0 & 2 & 3 & 6 & 4 & $\mathbf{3 , 8 0}$ & 1,03 & $27 \%$ \\
$\begin{array}{c}\text { Exhaustive } \\
\begin{array}{c}\text { Proche de la réalité interne } \\
\text { de l'entreprise agricole }\end{array}\end{array}$ & 0 & 3 & 2 & 4 & 6 & $\mathbf{3 , 8 7}$ & 1,41 & $31 \%$ \\
$\begin{array}{c}\text { Proche de la réalité externe } \\
\text { de l'entreprise agricole }\end{array}$ & 0 & 1 & 3 & 8 & 3 & $\mathbf{3 , 8 7}$ & 0,70 & $22 \%$ \\
$\begin{array}{c}\text { Proche de la réalité } \\
\text { de la gestion agricole }\end{array}$ & 0 & 1 & 6 & 3 & 5 & $\mathbf{3 , 8 0}$ & 1,07 & $27 \%$ \\
$\begin{array}{c}\text { Solution aux } \\
\text { problèmes actuels }\end{array}$ & 0 & 1 & 3 & 8 & 3 & $\mathbf{3 , 8 7}$ & 0,70 & $22 \%$ \\
$\begin{array}{c}\text { Atteinte des objectifs } \\
\text { du conseiller }\end{array}$ & 0 & 0 & 0 & 11 & 4 & $\mathbf{4 , 2 7}$ & 0,21 & $11 \%$ \\
$\begin{array}{c}\text { Atteinte des objectifs } \\
\text { de l'agriculteur }\end{array}$ & 0 & 0 & 2 & 9 & 4 & $\mathbf{4 , 1 3}$ & 0,41 & $15 \%$ \\
\hline
\end{tabular}

Note : L'évaluation des critères varie de pas du tout (1) à totalement (5).

elle tient compte des critères caractérisant l'environnement de la ferme. Mais, plus important encore, les conseillers ont trouvé qu'elle représente une bonne solution aux problèmes actuels. Enfin, les critères les plus élevés montrent que cette démarche permet d'atteindre les objectifs de l'agriculteur, comme ceux du conseiller.

Il faut également ajouter qu'en plus d'établir ces critères, la démarche a été bâtie de façon à séparer l'élaboration des stratégies de la gestion stratégique et à tenir compte autant de l'interne que de l'environnement, des objectifs du dirigeant que de la mission de l'entreprise.

\section{Les démarches pratiquées}

Lors des entrevues et à la suite de l'analyse de cas, il a été possible de découvrir que les conseillers, dans leur intervention auprès des agriculteurs, utilisent une démarche que l'on pourrait qualifier de standard. Globalement, la démarche utilisée ressemble à la gestion des opérations courantes pour optimiser le potentiel de l'entreprise (figure 3).

À l'aide de la revue de la documentation sur la gestion dans la PME, nous avons établi une liste des outils qui peuvent être utilisés pour rendre opérationnelle la démarche de gestion stratégique. Cette liste d'outils est présentée au tableau 3 de même que leur degré d'utilisation par les conseillers agricoles. Il a donc été possible de relever trois catégories d'outils. Tout d'abord, il y a les outils déjà 
Figure 3

La démarche adoptée par les conseillers agricoles

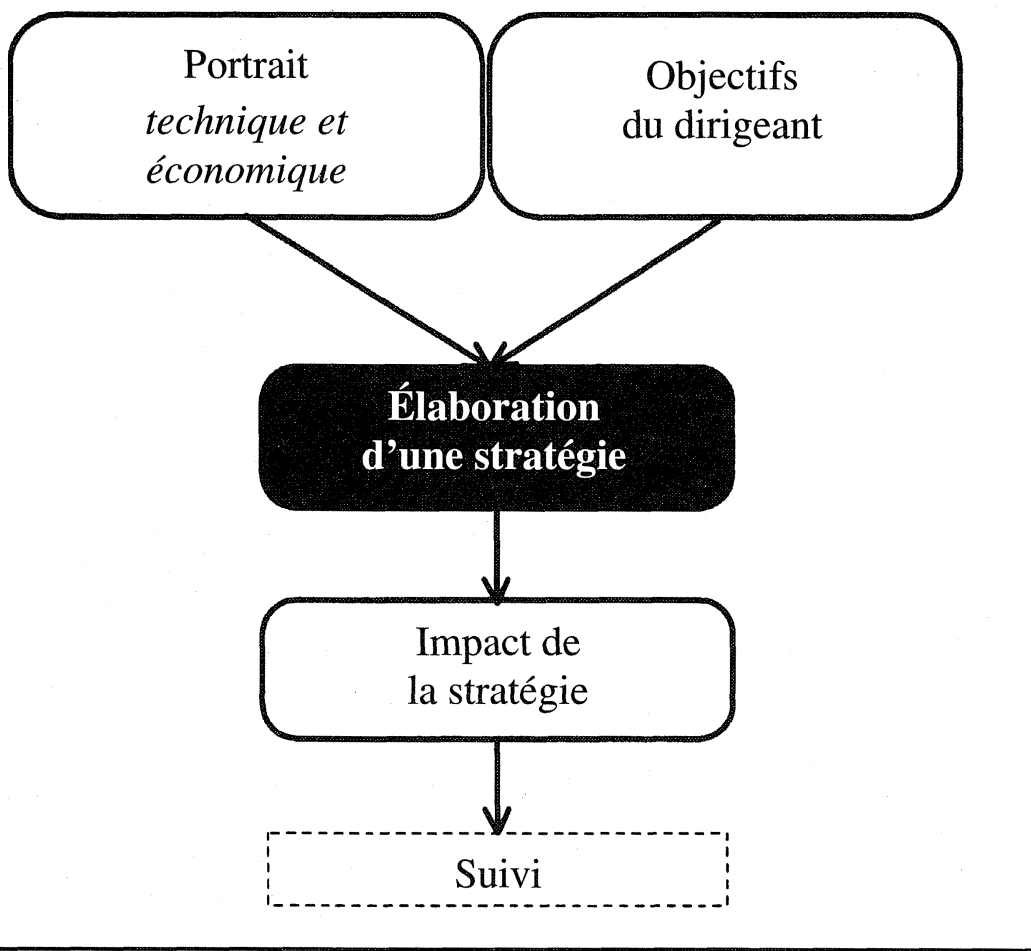

présents en agriculture (outils utilisés) et les outils non utilisés ou peu utilisés en gestion agricole. Le troisième groupe d'outils réunit ceux énumérés dans la revue de la documentation sur les PME, mais qui n'ont pas été mentionnés par les conseillers lors des entrevues.

Si l'on débute avec l'analyse interne, les conseillers possèdent l'essentiel des outils de base pour dresser le portrait de l'entreprise. Les outils supplémentaires, bien que non indispensables, permettent de raffiner l'analyse en apportant plus d'informations. Leur utilité est cependant à évaluer selon le type d'entreprise, car ils sont lourds à mettre en place et demandent beaucoup d'énergie. Il faut donc voir si l'information supplémentaire justifie un tel déploiement.

Selon le tableau 3, on décèle un manque flagrant d'outils d'analyse externe. Une fois encore, on peut dire qu' on dispose d'outils pour prendre connaissance de l'environnement de l'entreprise (journaux, colloques, conférences, etc.), mais pas pour l'analyser. Il est certain qu'ils ne sont peut-être pas utiles pour les productions 
TABleau 3

Classement des outils d'analyse

\begin{tabular}{|c|c|c|c|c|}
\hline $\begin{array}{l}\text { Étape d'utilisation } \\
\text { et } \\
\text { élément d'analyse }\end{array}$ & $\begin{array}{l}\text { Nom } \\
\text { de l'outil }\end{array}$ & $\begin{array}{l}\text { Utilisé } \\
\text { (au moins } \\
\text { un tiers } \\
\text { des } \\
\text { conseillers) }\end{array}$ & $\begin{array}{l}\text { Peu utilisé } \\
\text { (moins } \\
\text { d'un tiers } \\
\text { des } \\
\text { conseillers) }\end{array}$ & $\begin{array}{l}\text { Supplé- } \\
\text { mentaire } \\
\text { (jamais cité } \\
\text { par les } \\
\text { conseillers) }\end{array}$ \\
\hline
\end{tabular}

\section{Première phase : le diagnostic}

\begin{tabular}{|c|c|c|c|}
\hline Analyse interne & Découpe de l'entreprise & & $\checkmark$ \\
\hline \multirow[t]{8}{*}{ Analyse produit/service } & $\begin{array}{l}\text { Description qualitative } \\
\text { (supports écrits) }\end{array}$ & & $\checkmark$ \\
\hline & Comptabilité par activité & & $\checkmark$ \\
\hline & Chaîne de valeur & & $\checkmark$ \\
\hline & Courbe d'expérience & & $\sqrt{ }$ \\
\hline & États financiers ou comptables & $\checkmark$ & \\
\hline & $\begin{array}{l}\text { Références personnelles } \\
\text { (journaux...) }\end{array}$ & $\checkmark$ & \\
\hline & Critères techniques & $\checkmark$ & \\
\hline & Visites & 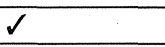 & \\
\hline \multirow[t]{6}{*}{$\begin{array}{l}\begin{array}{c}\text { Analyse des technologies, } \\
\text { de l'appareil de production }\end{array} \\
\end{array}$} & $\begin{array}{l}\text { Description qualitative } \\
\text { (supports écrits) }\end{array}$ & & $\checkmark$ \\
\hline & Efficacité & $\checkmark$ & \\
\hline & Coûts de production & $\checkmark$ & \\
\hline & Visites & $\checkmark$ & \\
\hline & Références personnelles & $\checkmark$ & \\
\hline & Ratios techniques & $\checkmark$ & \\
\hline \multirow[t]{5}{*}{ Analyse du personnel } & $\begin{array}{l}\text { Description qualitative } \\
\text { (supports écrits) }\end{array}$ & & $\checkmark$ \\
\hline & Références personnelles & $\checkmark$ & \\
\hline & États financiers & $\checkmark$ & \\
\hline & Ratios (UTP/hl) & $\checkmark$ & \\
\hline & Entretiens & $\checkmark$ & \\
\hline \multirow[t]{9}{*}{$\begin{array}{l}\text { Analyse comptable, financière } \\
\text { et économique }\end{array}$} & $\begin{array}{l}\text { Calcul des soldes } \\
\text { intermédiaires de gestion }\end{array}$ & & $\checkmark$ \\
\hline & Comptes de surplus & & $\checkmark$ \\
\hline & Analyse du fonds de roulement & & $\sqrt{ }$ \\
\hline & Tableau de financement & & $\checkmark$ \\
\hline & Entretiens & $\checkmark$ & \\
\hline & Budgets prévisionnels & $\checkmark$ & \\
\hline & Analyse de groupe & $\checkmark$ & \\
\hline & États financiers ou comptables & $\checkmark$ & \\
\hline & $\begin{array}{l}\text { Ratios économiques, } \\
\text { financiers et comptables }\end{array}$ & $\checkmark$ & \\
\hline \multirow[t]{2}{*}{ Analyse externe } & Segmentation de l'industrie & & $\checkmark$ \\
\hline & Description qualitative & & $\checkmark$ \\
\hline
\end{tabular}




\section{TABleau 3}

\section{Classement des outils d'analyse (suite)}

Analyse de l'environnement

\begin{tabular}{|c|c|c|c|}
\hline proche de l'entreprise & Analyse de la demande & & $\checkmark$ \\
\hline & Analyse de l'offre & & $\checkmark$ \\
\hline & Cycle de vie du produit & & $\checkmark$ \\
\hline & Analyse de portefeuille & & $\checkmark$ \\
\hline & Références personnelles & & \\
\hline & Entretiens & & \\
\hline \multirow[t]{5}{*}{$\begin{array}{l}\text { Analyse de l'environnement } \\
\text { éloigné de l'entreprise }\end{array}$} & Matrice d'attractivité & & $\checkmark$ \\
\hline & Analyse de la structure de marché & & $\sqrt{ }$ \\
\hline & Filières et réseaux & & $\checkmark$ \\
\hline & Arènes stratégiques & & $\sqrt{ }$ \\
\hline & Références personnelles & & \\
\hline \multirow[t]{3}{*}{$\begin{array}{l}\text { Définition des objectifs } \\
\text { et de la mission de l'entreprise }\end{array}$} & Plan d'affaires & $\checkmark$ & \\
\hline & $\begin{array}{l}\text { Document écrit } \\
\text { (moins formel que le précédent) }\end{array}$ & $\checkmark$ & \\
\hline & Entretiens & & \\
\hline \multicolumn{4}{|c|}{ Deuxième phase : la programmation } \\
\hline \multirow[t]{14}{*}{ Évaluation des stratégies } & $\begin{array}{l}\text { Méthodes économiques } \\
\text { (analyse de risque, MOTAD...) }\end{array}$ & & $\checkmark$ \\
\hline & $\begin{array}{l}\text { Techniques financières } \\
\text { (ratios, TIR, VAN, etc.) }\end{array}$ & & $\checkmark$ \\
\hline & Analyse de faisabilité & & $\checkmark$ \\
\hline & Entretiens & $\checkmark$ & \\
\hline & Documents pro forma & $\checkmark$ & \\
\hline & Élaboration de scénarios & $\checkmark$ & \\
\hline & $\begin{array}{l}\text { Avis des experts } \\
\text { (connaissances personnelles, etc.) } \checkmark\end{array}$ & & \\
\hline & Budget partiel & & \\
\hline & Budget global & & \\
\hline & Méthode Pert & & $\checkmark$ \\
\hline & Plan d'affaires & $\checkmark$ & \\
\hline & Entretiens & $\checkmark$ & \\
\hline & Calendrier & $\checkmark$ & \\
\hline & Budget & $\checkmark$ & \\
\hline \multicolumn{4}{|c|}{ Troisième phase : l'implantation } \\
\hline \multirow[t]{3}{*}{ Communication interne et externe } & Plan stratégique & & $\checkmark$ \\
\hline & Entretiens & $\checkmark$ & \\
\hline & Plan d'affaires & $\checkmark$ & \\
\hline \multirow[t]{2}{*}{ Veille stratégique } & Ratios et indicateurs & & $\checkmark$ \\
\hline & Connaissances personnelles & $\checkmark$ & \\
\hline \multirow[t]{4}{*}{ Contrôle et performance } & Plan stratégique & & $\checkmark$ \\
\hline & Analyse de groupe & $\checkmark$ & \\
\hline & Budgets & $\checkmark$ & \\
\hline & Critères techniques & $\checkmark$ & \\
\hline
\end{tabular}


contingentées et qu'ils sont longs à implanter, mais ils peuvent permettre de trouver de nouvelles solutions dans un contexte de libéralisation des marchés.

Enfin, il n'y a aucun outil nouveau pour l'analyse des objectifs : tous sont déjà connus. Mais il serait intéressant d'introduire plus de rigueur dans la définition des objectifs, entre autres, en les écrivant. Ainsi, l'utilisation d'un document doit continuer à être promue, par exemple, à travers celle du plan d'affaires.

Pour l'évaluation des stratégies (Phase II), les trois catégories d'outils sont représentées de façon égale, bien que chacune ait un objectif différent. Les outils utilisés servent surtout à évaluer l'impact financier de la stratégie sur l'entreprise (budgets partiels et globaux) ou à donner un avis qualitatif sur les stratégies (experts). Les outils peu utilisés peuvent servir à évaluer et à comparer différentes stratégies entre elles, sur le plan qualitatif (scénarios, entretiens), et les documents pro forma sont plus complets, mais demeurent du domaine financier. Les outils supplémentaires apportent une dimension statistique à l'évaluation et à la prise de décision (techniques économiques). Ils permettent une comparaison plus exacte de deux projets (analyse financière par TIR et VAN). Grâce à ces outils, il est plus facile de faire varier les hypothèses de base de chaque solution envisagée et d'en mesurer les conséquences sur la rentabilité de chaque projet de même que le risque inhérent.

Pour l'opérationalisation des stratégies, tous les outils sont connus, sauf la méthode Pert. Il y aurait donc lieu d'encourager leur utilisation. En effet, une plus grande rigueur dans l'opérationalisation accroît les chances de succès de la stratégie adoptée.

Il en va de même de la communication de la stratégie : les outils sont connus, mais peu utilisés. L'utilisation d'un plan d'affaires pour la communication externe de la stratégie facilite sa mise en œuvre. L'élaboration d'un plan stratégique, incluant non seulement le plan d'affaires, mais aussi les éléments de la décision et les résultats attendus, facilite la communication interne et la constitution de références une fois la stratégie mise en œuvre.

La veille stratégique reste un aspect à développer. En effet, les intervenants agricoles ne disposent pas d'outils pour surveiller l'environnement et repérer le moment propice pour appliquer une stratégie. Des indicateurs décrivant l'environnement doivent donc être établis, et ce, dans la mesure où plusieurs stratégies sont élaborées et que certaines seront déclenchées lorsque surviendra un événement donné.

Les outils de suivi (contrôle et performance) sont connus, mais encore une fois leur utilisation est peu fréquente, ce qui est dommage, car cela permettrait de suivre vraiment le déroulement de la stratégie. 


\section{Réactions des conseillers aux outils d'analyse}

Dans les étapes précédentes, les besoins des conseillers relatifs à une démarche complète de gestion stratégique ont été mis au jour. De même, leurs outils ainsi que des outils complémentaires ont été rattachés à chaque étape ou élément d'analyse de la démarche. «Vérifier l'adoption de la démarche et des outils d'analyse » constitue le dernier objectif des entrevues et de ce travail.

La plupart des conseillers ont réagi aux outils supplémentaires en affirmant qu'ils les utilisaient déjà. Toutefois, comme ces outils n'ont jamais été cités auparavant, nous pouvons en déduire qu'ils sont connus, mais pas vraiment utilisés. Les outils connus et jugés utiles dans la démarche sont à promouvoir, c'est-à-dire qu'il faut répandre leur utilisation.

Parmi les outils supplémentaires présentés au tableau 3, la chaîne de valeur, l'examen des coûts de production pour l'analyse des technologies, les états financiers pour l'analyse du personnel, les comptes de surplus et les budgets partiels pour l'analyse financière ont été jugés inutiles par les conseillers. À part ces derniers, les gestionnaires agricoles jugent que cette démarche et ses outils d'analyse sont susceptibles d'avoir un impact positif sur la gestion, la rentabilité et la compétitivité de l'entreprise agricole sur ses marchés nationaux et internationaux. Cet impact prévisible s'explique, selon eux, par la rigueur qu' apporte une telle démarche dans l'élaboration des stratégies. En effet, cette démarche structure la réflexion, fait prendre conscience des capacités et des potentialités de l'entreprise dans son environnement, guide mieux les choix et augmente le contrôle; autant de fonctions qui limitent l'emprise du hasard, diminuent les risques et augmentent les chances de succès, donc la rentabilité de l'entreprise.

Cet enthousiasme pour la démarche amène les conseillers à souhaiter son implantation dans l'entreprise agricole. Mais, à ce niveau, il convient de séparer les gestionnaires en deux groupes. Si tous souhaitent sa mise en œuvre, tous ne veulent pas s'en charger eux-mêmes. En effet, selon leur profession, certains veulent l'appliquer, d'autres la faire adopter par les agriculteurs. Les conseillers en financement (institutions privées, coopérative, sociétés de financement et de crédit agricole) sont favorables à l'implantation de cette démarche, car elle faciliterait leur travail pour évaluer un dossier pour l'accord d'un financement. Mais ils ne peuvent pas l'entreprendre à la place de l'agriculteur; ils n'en ont ni le temps, ni la mission. Et s'ils le faisaient, en tant qu'évaluateurs de projet, ils deviendraient juge et partie. À l'opposé, les conseillers de gestion (SGA, MAPAQ) sont prêts à l'entreprendre. Ils considèrent en faire déjà une bonne partie et être à même de la compléter avec l'agriculteur. L'UPA se situe un peu en marge de cette seconde catégorie. En effet, si ses conseillers jugent la démarche pertinente, ils estiment qu'ils auraient du mal à la mettre en œuvre, car leurs services doivent être rémunérés par l'agriculteur et ce dernier ne serait pas prêt à débourser la somme qu'une telle démarche exigerait. 
Si nous résumons, les entrevues montrent que la démarche peut être adoptée par les gestionnaires agricoles, que les conseillers de financement promeuvent son implantation et que les conseillers de gestion sont prêts à la mettre en place. Il reste cependant une grande inconnue : la réaction des agriculteurs face à cette démarche. Aucune réponse ne peut être apportée à cette interrogation dans le cadre de cette recherche. Toutefois, les conseillers ont suggéré différentes formes d'implantation pour sensibiliser les agriculteurs. Elles représentent les premiers pas vers la sensibilisation des agriculteurs à cette démarche de gestion stratégique et, donc, vers son adoption.

Les conseillers interrogés estiment que le meilleur outil de sensibilisation des agriculteurs demeure l'utilisation d'exemples. En effet, on ne peut pas atteindre les agriculteurs en présentant uniquement des concepts. Beaucoup de discours ont déjà été faits aux agriculteurs dans ce sens et beaucoup de démarches ont été proposées sans aboutir à quoi que ce soit. L'implantation sur quelques fermes, puis la présentation des résultats obtenus par la démarche restent les meilleurs vecteurs de diffusion. Le choix de ces fermes ne devrait pas poser de problèmes selon les conseillers. En effet, certaines fermes sont déjà prêtes à entreprendre ce type de démarche, et ce, pour n'importe quel type de production. Si la démarche réussit chez quelques producteurs, leaders dans leur domaine, les autres seront enclins à emboîter le pas.

Par ailleurs, l'utilisation d'un guide a été suggérée par certains conseillers. Toutefois, cette forme de diffusion est plus discutable dans la mesure où certains estiment que les agriculteurs ont déjà beaucoup de «paperasse » à lire. Ce guide devrait être le plus concis possible. Ainsi, il ne pourrait présenter que la démarche elle-même et ses étapes, pour ne pas l'alourdir avec la liste de tous les outils d'analyse. Toutefois, en ne présentant que la démarche, ce guide risquerait de ne pas être accepté par les agriculteurs, eu égard à ce qui a été dit précédemment. Il s'adresserait donc plus particulièrement aux conseillers pour lesquels la présentation des outils est inutile. En effet, ils possèdent déjà certains outils et, si besoin est, ils peuvent aller chercher ceux qui leur manquent. L'ajout des outils au guide n'encouragerait ni à le lire, ni, a fortiori, à adopter la démarche qu'il propose.

\section{Conclusion}

Notre première question de recherche visait à identifier les pratiques utilisées actuellement en agriculture et à déterminer si elles s'inséreraient dans une démarche d'analyse stratégique. Cette question nous a d'abord conduits à concevoir une démarche d'analyse stratégique adaptée à l'agriculture, ce qui a fourni un modèle de référence pour juger des pratiques actuelles en analyse stratégique. Le second objectif était de recenser les pratiques de gestion agricole et les besoins des gestionnaires agricoles pour élaborer la stratégie d'entreprise, ce qui fut fait. La démarche actuelle d'analyse stratégique des conseillers a été schématisée et sa comparaison 
avec le modèle de gestion stratégique a fait ressortir des besoins en analyse externe, comparaison des stratégies, élaboration des opérations, communication, veille stratégique et suivi. Le recensement des outils utilisés actuellement ou envisagés pour réaliser la démarche de gestion stratégique a également permis de relever des besoins en outils manquants ou en outils complémentaires. Après quoi, pour répondre à la première question de recherche, nous pouvons affirmer que les pratiques actuelles ne suffisent pas à implanter une démarche complète de gestion stratégique.

Nous avons tenté de répondre à notre deuxième question de recherche : «Peuton adapter les outils utilisés dans les PME des autres secteurs économiques pour compléter la démarche d'analyse stratégique dans le contexte particulier des exploitations agricoles ? » Cette question nous a amenés à « recenser les outils d'analyse stratégique utilisés par les PME». À partir de ce recensement, nous avons dressé une liste d'outils pertinents à la démarche de gestion stratégique. Cette liste comprend les outils utilisés actuellement en agriculture, ceux qui sont peu utilisés et des outils supplémentaires issus des PME.

Finalement, nous avons voulu savoir si la démarche et les outils identifiés seront adoptés par les gestionnaires agricoles. L'étude démontre en effet que la démarche de gestion stratégique élaborée et ses outils d'analyse pourraient être adoptés par les intervenants en gestion agricole.

\section{Bibliographie}

ATTONATY, J.M. et L.G. SOLER (1992), «Aide à la décision et gestion stratégique : un modèle pour l'entreprise agricole », Revue Française de Gestion, n ${ }^{\circ} 88$, mars-avril.

D’Amboise, G. (1986), Gestion stratégique des P.M.E. : des modèles émergents de la littérature, Sainte-Foy, Québec, Université Laval, Faculté des sciences de l'administration.

D’AmвoISE, G. (1994), Strategic Planning in SMEs : A Synthesis of Research Results, Sainte-Foy, Québec, Université Laval, Faculté des sciences de l'administration, 64 pages.

HARLING, K.F. (1992), «A test of the applicability of strategic management to farm management », Canadian Journal of Agricultural Economics.

LAMBERT, R., P. CALKInS et R. Michaud (1992), Outils de gestion d'aide à la prise de décision dans le secteur horticole du Québec, Série Recherche no 18, Sainte-Foy, Québec, Université Laval, Département d'économie rurale, Groupe de recherche agroalimentaire (GRAAL), août.

LAMBERT, R., R. ROMAIN, S. MAISONNAS et C. ROY (1995), L'analyse stratégique et ses outils de gestion: Théorie et application dans l'entreprise agricole, Série Recherche $\mathrm{n}^{\circ} 35$, Sainte-Foy, Québec, Université Laval, Département d'économie rurale, Groupe de recherche agroalimentaire (GRAAL), septembre. 
MintzBerg, H. (1994), Grandeur et décadence de la planification stratégique, Paris, Dunod.

WESTGREN, R.E., S.T. SONKA et K.K. LITZENBERG (1988), « Strategic issue identification among agribusiness firms », Agribusiness, vol. 4, $\mathrm{n}^{\circ} 1$. 